\title{
Pierre Lamard et Nicolas Stoskopf (dir.), La transition énergétique, un concept historique?
}

Villeneuve-d'Ascq, Presses universitaires du Septentrion, 2018

\section{François Jarrige}

\section{(2) OpenEdition}

\section{Journals}

Édition électronique

URL : http://journals.openedition.org/artefact/4382

DOI : $10.4000 /$ artefact. 4382

ISSN : 2606-9245

\section{Éditeur :}

Association Artefact. Techniques histoire et sciences humaines, Presses universitaires du Midi

\section{Édition imprimée}

Date de publication : 15 octobre 2019

Pagination : 255-260

ISBN : 978-2-8107-0650-1

ISSN : 2273-0753

\section{Référence électronique}

François Jarrige, «Pierre Lamard et Nicolas Stoskopf (dir.), La transition énergétique, un concept historique? », Artefact [En ligne], 10 | 2019, mis en ligne le 06 août 2020, consulté le 27 novembre 2020. URL : http://journals.openedition.org/artefact/4382 ; DOI : https://doi.org/10.4000/artefact 4382

Ce document a été généré automatiquement le 27 novembre 2020.

\section{cc)}

Artefact, Techniques, histoire et sciences humaines est mise à disposition selon les termes de la Licence Creative Commons Attribution - Pas d'Utilisation Commerciale - Pas de Modification 4.0 International. 


\section{Pierre Lamard et Nicolas Stoskopf (dir.), La transition énergétique,} un concept historique?

Villeneuve-d'Ascq, Presses universitaires du Septentrion, 2018

\section{François Jarrige}

\section{RÉFÉRENCE}

Pierre Lamard et Nicolas Stoskopf (dir.), La transition énergétique, un concept historique?, Villeneuve-d'Ascq, Presses universitaires du Septentrion, 2018, 322 p.

1 Cet ouvrage collectif dirigé par les historiens Pierre Lamard et Nicolas Stoskopf est la publication des actes des sixièmes journées d'histoire industrielle organisées en 2014 par l'Université de Haute Alsace et l'Université de Technologie de Belfort-Montbéliard. Il aborde de front la question essentielle des transitions énergétiques, devenue centrale dans les arènes publiques comme dans les sciences sociales contemporaines. Nous assistons en effet au retour massif de la "question énergétique » en ce début du XXI siècle, porté notamment par l'urgence climatique et les risques associés aux infrastructures énergétiques contemporaines. La transition énergétique est désormais au cœur des politiques publiques, elle provoque de vives controverses sociotechniques, et l'ouvrage cherche à prendre de la hauteur à l'égard de ces urgences en revenant à l'histoire. L'enjeu est d'abord d'interroger cette catégorie de "transition énergétique " au prisme du passé. Le point d'interrogation qui suit le titre suggère une approche réflexive et critique à l'égard d'une telle notion à la fois vague, polysémique, portée par des politiques publiques et des industriels défendant leur propre agenda. Il s'agit d'explorer ses usages possibles en histoire, mais aussi ses limites comme catégorie réellement heuristique.

On regrettera peut-être que l'ouvrage n'interroge pas davantage cette catégorie de « transition » qui est pourtant au cœur de l'analyse, même si on trouve bien entendu de 
nombreux éléments de discussion au fil de la lecture. Il aurait été utile de suivre sa généalogie intellectuelle, ses usages et fonctions aujourd'hui comme hier, puisque ce sont eux qui en définissent largement les significations. Comme l'a suggéré JeanBaptiste Fressoz, l'idée de transition appliquée à la question de l'énergie trouve en effet ses racines dans le contexte des «crises» des années 1970 et dans la promotion de nouvelles politiques publiques censées conjurer les difficultés que traversent alors les sociétés industrielles en Europe et aux États-Unis. La notion serait apparue au sein des institutions internationales et des groupes d'intérêts soucieux de construire des images moins anxiogènes du futur, elle aurait été forgée par des think tanks et popularisée par de grandes institutions publiques comme le «Bureau de la planification énergétique américain». Le recours à l'expression "transition énergétique» aurait servi de substitut à celle de "crise énergétique ", en incitant à l'adoption de technologies et d'énergies alternatives au pétrole. Mais l'idée de transition pose problème dans la mesure où elle réactive une vision linéaire du temps historique, là où dominaient souvent la pluralité et la coexistence de systèmes adaptés localement aux ressources disponibles, comme aux contraintes sociales et environnementales. L'idée de transition pose également problème car elle véhicule un grand récit progressiste alors que l'histoire de l'énergie est bien plus celle d'additions successives que de transitions.

3 Les trois parties qui structurent le volume s'intitulent «Retour sur des temporalités particulières », « des récurrences et des échecs » et " des transitions, des contraintes ». Peut-être une organisation chronologique aurait-elle permis un traitement plus clair et offert une vision plus synthétique des évolutions, en montrant les temps forts que représentent par exemple l'entre-deux-guerres ou la décennie 1970. Peut-être d'autres regroupements thématiques auraient-ils été mieux à même d'articuler les riches études de cas réunies dans l'ouvrage. Par ailleurs, et contrairement à ce que laisse entendre le titre, l'ouvrage porte surtout sur le $\mathrm{xx}^{\mathrm{e}}$ siècle, en dehors de l'intéressant texte de Pierre Fluck sur l'hydraulique depuis l'Antiquité. Il y rappelle combien l'énergie hydraulique "prétendument vieillotte» n'a cessé d'être réinventée et adaptée à de nouveaux usages, comme l'avait montré Serge Benoit, malheureusement non cité, dans son travail sur la «modernité de la tradition » hydraulique. Or le $\mathrm{xx}^{\mathrm{e}}$ siècle représente un moment de très forte croissance de la demande et de la consommation énergétique elle est multipliée par douze - et il possède d'importantes singularités qui auraient pu être mieux soulignées et questionnées en assumant le choix de se concentrer sur cette période d'imposition massive des sources d'énergies fossiles et de transformation des anciens systèmes énergétiques.

4 Mais au-delà de ces quelques remarques il faut d'abord saluer l'ampleur du travail réalisé, la diversité des thématiques abordées, la richesse des diverses contributions, qui offrent de passionnants essais sur quelques-uns des enjeux centraux posés par le sujet et qui se répondent souvent dans un dialogue constructif et pertinent. La restitution de cette richesse s'avère délicate mais on peut la tenter en distinguant par exemple les principaux types de carburants et les principales sources d'énergie primaires examinées dans le volume. Commençons par les énergies fossiles, celles qui s'imposent massivement à l'époque contemporaine, en remodelant en profondeur les économies et les sociétés. Le pétrole est assez peu présent, ce qui peut étonner si on considère son rôle décisif au cours $\mathrm{du} \mathrm{xx}^{\mathrm{e}}$ siècle et le nombre croissant de travaux notamment anglo-américains - sur ce carburant liquide. On le trouve principalement évoqué dans la contribution d'Aymen Boughanmi sur la conversion de la marine 
britannique au pétrole au début du $\mathrm{xx}^{\mathrm{e}}$ siècle, à travers l'action de l'amiral Fisher replacé dans une réflexion globale sur les enjeux impériaux que posait cette substitution. Le charbon en revanche est bien davantage présent, témoignant de l'ancienneté et de l'importance de l'historiographie européenne de la mine et des mineurs. Nadège Sougy explore ainsi les stratégies de valorisation des houilles pendant l'entre-deux-guerres et les efforts de classifications mis en place pour tenter de rationaliser et économiser cette ressource dont on sait que la France manque cruellement. Jean-Louis Escudier étudie la période qui suit en présentant les diverses stratégies employées après 1945 par les Charbonnages de France pour maintenir, voire relancer, l'exploitation de la houille malgré le contexte de crise qui aboutit finalement à l'arrêt de l'exploitation.

5 Le nucléaire est également, et de façon logique, très présent, en particulier avec deux contributions qui lui sont spécifiquement consacrées et qui ouvrent le volume. Yves Bouvier revient sur les discours tenus sur l'avenir de l'énergie nucléaire en France après 1945. Il montre très bien les registres de justifications avancés pour accompagner cette transition "par le haut", si caractéristique de la situation française. Il est par exemple intéressant de se rappeler comment l'énergie nucléaire fut présentée au cours des années 1950 par certains scientifiques - et contre l'avis déjà convaincu des responsables administratifs et politiques - comme une énergie de transition, c'est-àdire nécessairement provisoire, et destinée à préparer un avenir solaire et renouvelable! L'auteur a par ailleurs pleinement raison de rappeler que l'étude de l'évolution des systèmes énergétiques impose de prendre en considération l'ensemble des dimensions - politiques, économiques, imaginaires - du processus et pas « la seule étude des discours technocritiques» (p.38), encore convient-il de ne pas totalement évacuer cet aspect comme c'est souvent le cas, et de ne pas laisser de côté les travaux sur les travailleurs du nucléaire, qu'ils soient ici ou en Afrique. La contribution de Robert Belot envisage ensuite l'énergie nucléaire comme une « figure épiphanique de la science agissante » après 1945. Dans la continuité de son ouvrage précédent «L'atome et la France ", il propose une réflexion sur l'imaginaire de la nouvelle énergie dans l'après-guerre. Sa thèse est que la nucléarisation de la France relève d'abord d'une attente quasi révolutionnaire et eschatologique dans l'avènement d'un monde nouveau, "voire d'une révélation, d'ordre quasiment épiphanique, qui témoignerait de la capacité de l'homme, après l'obscurantisme nazi et la régression vichyste, à maîtriser le réel [...] et à œuvrer pour le progrès de l'humanité »(p.40). Cette réflexion aide à comprendre pourquoi les anciens doutes sur le progrès technique, très vifs avant la guerre, ne renaissent pas après 1945 et comment a pu s'imposer la nouvelle trajectoire nucléaire en dépit des immenses incertitudes et risques qu'elle introduisait.

6 Les énergies renouvelables sont également présentes, ce sont autour d'elles que se structurent et se réinventent sans cesse les discours et pratiques de transition. Ces questions, longtemps laissées de côté, bénéficient d'un intérêt soutenu aujourd'hui comme le montrent en particulier les contributions d'Anaël Marec et Philippe Bruyerre dans la continuité de leurs recherches doctorales récentes. La première propose ainsi une intéressante réflexion sur « les îles industrielles » et les utopies qui les portent, en comparant deux projets de centrales offshore - l'un des années 1920, l'autre développée aujourd'hui dans la région Pays de La Loire - fonctionnant via les énergies marines, locales et "renouvelables ». Le second revient sur l'électrification rurale au Danemark au début $\mathrm{du} \mathrm{xx}^{\mathrm{e}}$ siècle via les premières éoliennes électriques mises au point par le célèbre ingénieur Poul La Cour. Paul Naegel présente de son côté la célèbre expérience 
de l'usine marémotrice de la Rance, et Alexandre Giandou revient sur la genèse de la "Compagnie nationale du Rhône " pensée comme "modèle pour la transition ». On voit combien les énergies "naturelles» devenues «renouvelables» n'ont cessé de nourrir de multiples projets et expérimentations, dont beaucoup restent encore à exhumer.

7 D'autres contributions se concentrent sur un pays ou une région pour reconstituer leurs dynamiques propres, à l'image du texte de Serge Pasquier sur le passage du bois au charbon, puis à l'hydroélectricité en Suisse des années 1850 aux années 1950 ; ou celui de Régis Boulat sur les efforts des industriels alsaciens pour réduire leurs dépenses d'énergie, et ainsi leur coût, après 1973. Il est intéressant d'apprendre que de notables baisses de consommations ont lieu dans les entreprises alsaciennes à l'époque : à Peugeot 36,6 \% d'économie sont réalisées entre 1973 et 1979, mais aussi que, refusant d'envisager réellement une restriction de la demande, le patronat alsacien s'est engagé dans une intense "propagande » en faveur de l'énergie nucléaire. On peut intégrer dans le même ensemble l'intéressante réflexion d'Arnaud Peters et Olivier Defêchereux sur les évolutions énergétiques dans le bassin industriel liégeois de l'entre-deuxguerres, saisies à travers les archives des établissements industriels, insalubres et dangereux.

8 Un dernier ensemble de textes explore des projets qui ont échoué, à l'image de la voiture électrique étudiée par Jean-Louis Loubet. L'auteur rappelle le retour régulier des promesses de voitures électriques tout au long du xx siècle: déjà en 1899 le Touring Club de France s'enthousiasmait pour la double motorisation « du pétrole et de l'électricité " présentée comme "l'automobilisme de l'avenir ", et en $190430 \%$ des automobiles américaines étaient électriques. L'essor puis l'enfouissement des projets de voitures électriques tiennent à des facteurs complexes: la faiblesse du marché, les freins culturels, et les améliorations trop limitées en matière de stockage de l'énergie, autant de contraintes qui devraient être levées selon l'auteur au xxI siècle puisqu'il annonce le « succès probable demain » de la voiture électrique (p. 98). Un autre cas très intéressant concerne les "carburants de remplacement» que furent l'alcool et les gazogènes dont l'histoire, reconstituée par Camille Molles, offre de nombreux éléments éclairants sur les difficultés rencontrées par les tentatives pour substituer une source d'énergie à une autre.

9 Ces quelques mots ne suffisent évidemment pas à rendre compte de la richesse de ces quinze études de cas qui montrent comment les sociétés du $\mathrm{xx}^{\mathrm{e}}$ siècle n'ont cessé d'être travaillé par les débats sur l'énergie, leurs avantages respectifs et leurs faiblesses, mais aussi les nécessaires transitions et substitutions d'un système technique et d'un carburant à l'autre. Les contributions portent surtout sur les innovations technologiques et sur la France, la question d'une baisse des consommations énergétiques et les dynamiques spatiales plus globales sont en revanche peu abordées alors qu'elles pourraient apparaitre centrales pour engager une réelle transition dans nos imaginaires et nos systèmes socioénergétique. Pourquoi faudrait-il par ailleurs que l'essor des consommations énergétiques soit considéré comme l'étalon de mesure du devenir des sociétés ? Il reste par ailleurs encore beaucoup de travaux à mener pour écrire cette histoire en s'émancipant des mythes et des idéologies qui pèsent ici plus que dans n'importe quel domaine de l'histoire. Cet ouvrage y contribue en proposant un très utile état des lieux des recherches menées en France sur le sujet, en dessinant 
de nombreuses pistes et en posant un certain nombre de débats essentiels pour l'avenir.

\section{AUTEURS}

\section{FRANÇOIS JARRIGE}

Université de Bourgogne, Centre Georges-Chevrier (UMR 7366) 